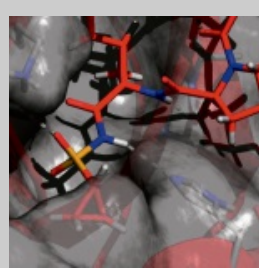

\title{
Medicinal Chemistry and Chemical Biology Highlights
}

Division of Medicinal Chemistry and Chemical Biology

\section{Tumor Targeting with Small Molecule-Drug Conjug- ates (SMDCs) - Can They be Better than ADCs?}

\section{Karl-Heinz Altmann}

${ }^{*}$ Correspondence: Prof. K.-H. Altmann, ETH Zurich, Department of Chemistry and Applied Biosciences, $\mathrm{HCl} \mathrm{H405,} \mathrm{Vladimir-Prelog-Weg} \mathrm{4,} \mathrm{CH-8093} \mathrm{Zurich,} \mathrm{E-mail:}$

karl-heinz.altmann@pharma.ethz.ch

\section{Keywords: Antibody-drug conjugates · Anticancer drug} discovery · Small molecule-drug conjugates · Tumor targeting · Tumor uptake

Over the last three decades, anticancer drug discovery and development have undergone a profound shift in focus from classical chemotherapeutics to targeted agents with significantly enhanced potential for tumor specificity and improved side effect profiles. This has included, e.g. small molecules or protein therapeutics acting on different aspects of derailed signaling pathways in cancer cells or, more recently, interfering with deregulated epigenetic modulators. Alternatively, strategies have been developed by which highly potent cytotoxic agents are rendered (more) tumor-specific by means of an appended targeting moiety that interacts with a protein unique to or at least significantly more abundant on tumor cell surfaces than on normal cells. Currently, the most important and successful targeting moieties in use are monoclonal antibodies; ${ }^{[1]}$ four antibody-drug conjugates (ADCs) have received marketing authorization for different types of cancers and many more are in clinical development. ${ }^{[2,3]}$ For the vast majority of ADCs, binding to a cell surface receptor results in internalization of the construct and subsequent intracellular drug release by different mechanisms. However, it has been shown that ADCs binding to a non-internalizing target can also exert very potent antitumor activity in animal models. ${ }^{[4]}$ Although monoclonal antibodies exhibit exquisite specificity for their protein targets, ADCs, quite intriguingly, are not free of side effects. While the reasons for this observation have not been fully elucidated, one principle issue associated with all protein-based cancer therapeutics is their limited ability to extravasate and penetrate deep into a solid tumor mass. ${ }^{[5]}$ In addition, the long circulatory half-life of ADCs may lead to toxicity due to continuous premature drug cleavage.

Compared to ADCs, constructs that rely on small molecules, rather than proteins, as tumor-targeting moieties should penetrate solid tumors much more efficiently. In fact, small molecule-drug conjugates (SMDCs) have been explored extensively as potential anticancer agents; ${ }^{[6,7]}$ however, while several SMDCs have been and are also currently evaluated in clinical trials, no SMDC has yet gained marketing approval. Among the limited number of targets that have been addressed by SMDCs so far, the most prominent is the folate receptor. A vinblastine-folate conjugate (vintafolide) has been advanced to phase III clinical trials, ${ }^{[6]}$ but these trials have been terminated for undisclosed reasons.

Recently, the group of Dario Neri at the ETH Zürich has described the SMDC AAZ-MMAE ${ }^{[8]}$ (Fig. 1) that is derived from the known carbonic anhydrase IX (CAIX) inhibitor acetazolamide

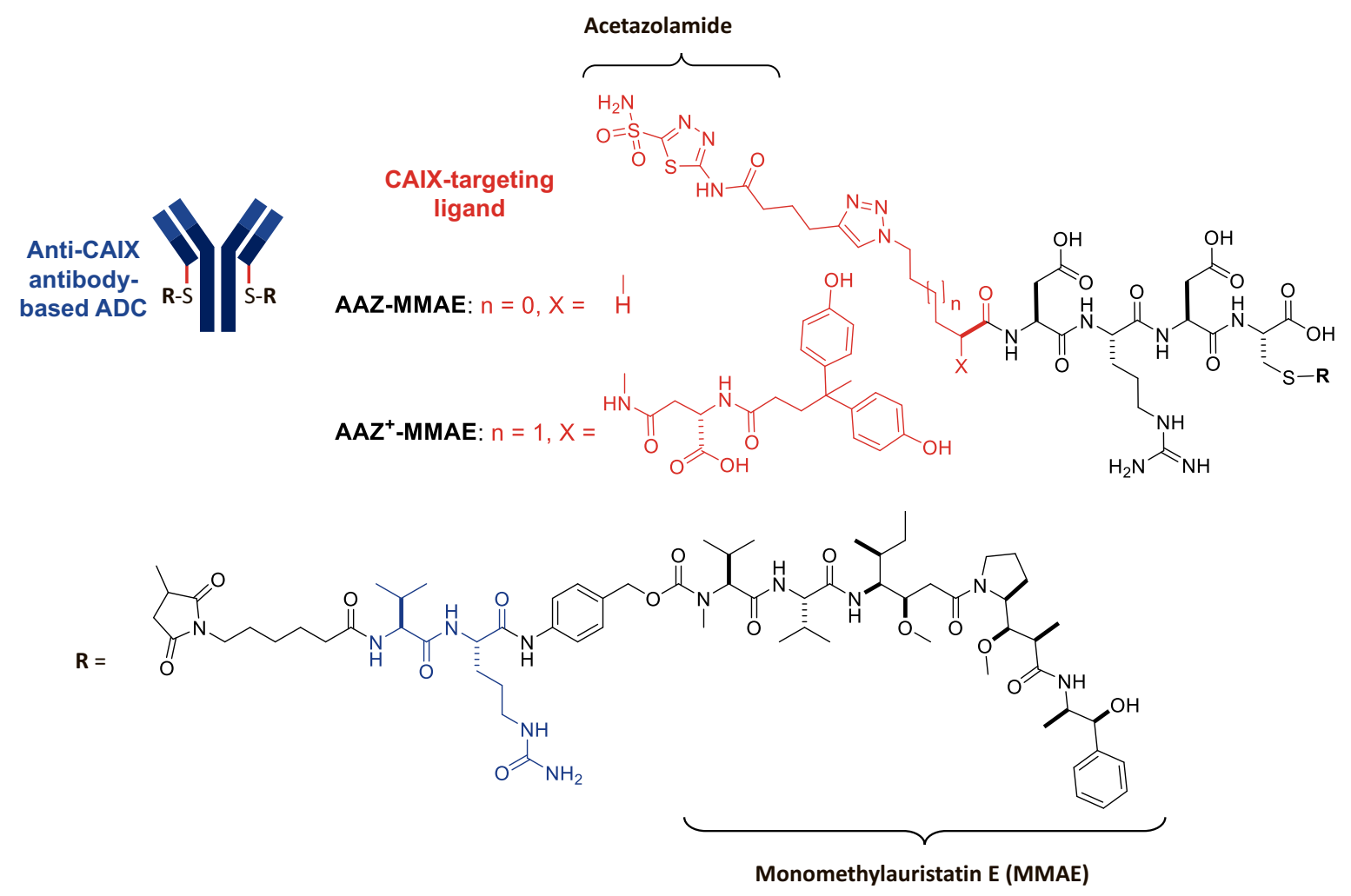

Fig. 1. Structures of CAIX-targeted SMDCs and an ADC. 
(AAZ) as the tumor-targeting moiety and incorporates the tubulin inhibitor monomethyl auristatin E (MMAE) as the cytotoxic payload. The linker moiety connecting the two parts includes a cleavable valine-citrulline (Val-Cit) dipeptide unit and a selfimmolative element, which are also part of the linker moiety in the approved ADC brentuximab vedotin (Adcetris ${ }^{\circledR}$ ). CAIX is a cell membrane protein that is overexpressed on a multitude of cancers and thus represents an attractive target for either ADCs or SMDCs. While CAIX is not internalized upon ligand binding, AAZ-MMAE nevertheless produced profound antitumor effects in mouse models at well tolerated doses, thus indicating that tumor-specific linker cleavage can also occur in the extracellular environment. ${ }^{[8]}$

In their most recent study, which is the very first of its type, Neri and co-workers have now compared the tumor accumulation and the in vivo efficacy of CAIX-targeted ADCs and SMDCs. ${ }^{[9]}$ Employing a new acetazolamide-derived, very high affinity CAIX ligand $\left(\mathbf{A} \mathbf{A Z}{ }^{+}\right)$that they had retrieved from a DNAencoded library, they have prepared the CAIX-targeted SMDC AAZ + -MMAE, which binds to CAIX with a $\mathrm{K}_{\mathrm{d}}$ of $10 \mathrm{nM}$. A corresponding CAIX-directed ADC was obtained from the monoclonal anti-CAIX XE114 antibody ( $\mathrm{K}_{\mathrm{d}}$ of the ADC for CAIX, $0.13 \mathrm{nM}$ ). As demonstrated with a radioactively labelled derivative of the low-molecular weight ligand and a ${ }^{125} \mathrm{I}$-labelled version of the antibody, the SMDC ligand displayed a vastly superior tumor to blood ratio (Fig. 2) and substantially higher tumor uptake ( $c a .40 \%$ of the injected dose (ID)/g of tumor 6 $\mathrm{h}$ after injection). In addition, fluorescence microscopy studies revealed homogenous uptake of the SMDC ligand across the tumor mass after $1 \mathrm{~h}$, while the antibody showed only patchy perivascular uptake in tumor cells.

Both $\mathbf{A} \mathbf{A Z} \mathbf{Z}^{+}$-MMAE and the corresponding ADC exhibited potent antitumor activity in mice bearing human SKRC-52 kidney tumors, although neither agent produced lasting regressions (Fig. 3). Somewhat surprisingly, the in vivo antitumor activity of AAZ ${ }^{+}$-MMAE was slightly inferior to that of the ADC, in spite of its substantially more favorable tumor uptake. The reasons for this finding are not entirely clear at this point, but Neri and co-workers hypothesize that the activity of the ADC is in fact based on the slow release of the highly potent MMAE payload. This hypothesis is line with the observation that an analogous control ADC based on the KSF antibody, which is specific for hen egg lysozyme and hence does not target tumor cells, also displayed potent antitumor activity in the SKRC-52 model. At the same time, it may be possible to improve the in vivo potency of AAZ ${ }^{+}$-MMAE by careful fine-tuning of the stability of the linker moiety between the targeting ligand and the MMAE payload, as suggested by Neri and co-workers. Indeed, given the fact that AAZ ${ }^{+}$-MMAE exhibits a tumor to blood ratio of 100:1 only $6 \mathrm{~h}$ after $i$. v. injection, it is well conceivable that a more labile linker could lead to improved antitumor activity.

These questions will have to be addressed in future studies. Independent of this, however, the results reported by Neri and coworkers clearly show that SMDCs can outcompete ADCs with regard to general biodistribution and tumor uptake. At the same time, the data reconfirm that SMDCs that are not internalized upon binding to a tumor cell surface protein can exhibit potent antitumor activity. Given the rapid evolution of technologies that allow for the identification of high-affinity low molecular weight ligands for a given protein target, e.g. based on DNA-encoded libraries, the SMDC approach holds significant promise for the development of novel, efficacious cancer therapies.

Received: February 6, 2018

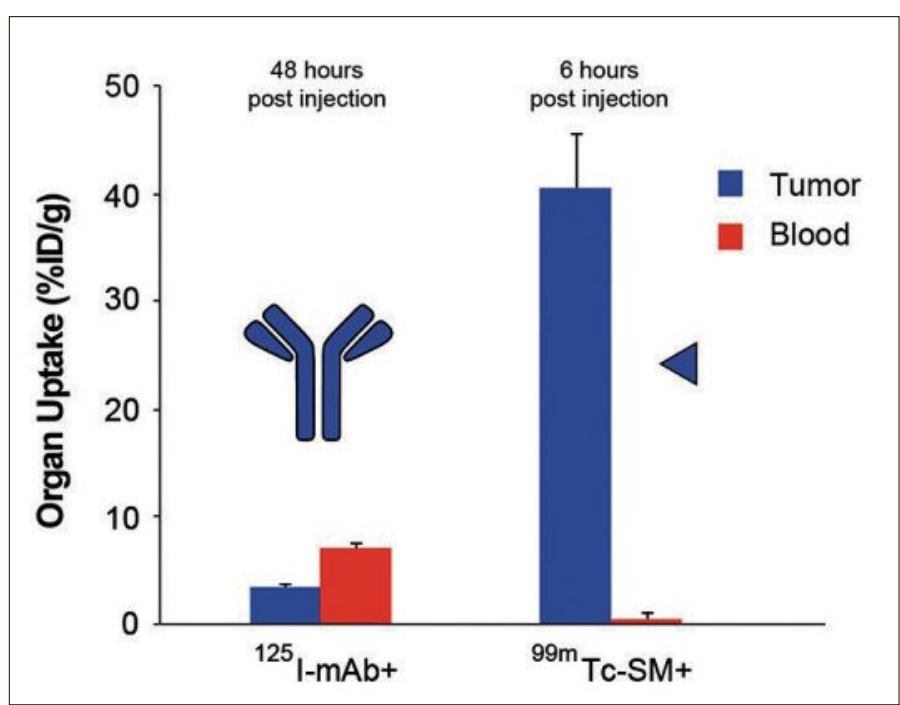

Fig. 2. Tumor-targeting performance of the anti-CAIX XE114 antibody $(\mathrm{mAb}+)$ and the small ligand $\mathrm{AAZ}+(\mathrm{SM}+)$ against human renal cell carcinoma cells SKRC-52 xenografted in mice. ID = injected dose. Reprinted with permission from S. Cazzamalli, A. Dal Corso, F. Widmayer, D. Neri. J. Am. Chem. Soc. 2018, ASAP, http://dx.doi. org/10.1021/jacs.7b13361. Copyright 2018 American Chemical Society.

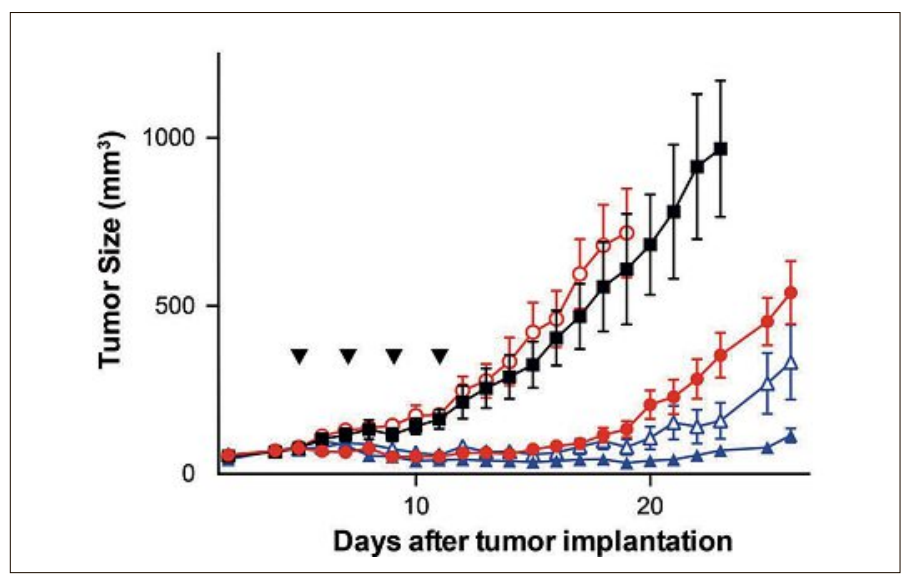

Fig. 3. Antitumor activity of the anti-CAIX antibody-drug conjugate and of $A A Z^{+}-M M A E$ in BALB/c nu/nu mice bearing SKRC-52 xenografts. Filled red circles: $\mathbf{A A Z}-\mathbf{M M A E}, 250 \mathrm{nmol} / \mathrm{kg}$; open red circles: control SMDC without acetazolamide targeting group, $250 \mathrm{nmol} / \mathrm{kg}$; filled blue triangles: CAIX-targeted ADC, $125 \mathrm{nmol} / \mathrm{kg}$; open blue triangles: nontargeted ADC, $125 \mathrm{nmol} / \mathrm{kg}$; black rectangles: PBS. The arrows indicate intravenous (i.v.) administration of the corresponding agent. Reprinted from S. Cazzamalli, A. Dal Corso, F. Widmayer, D. Neri. J. Am. Chem. Soc. 2018, ASAP, http://dx.doi.org/10.1021/jacs.7b13361. Copyright 2018 American Chemical Society.

[1] R. V. Chari, M. L. Miller, W. C. Widdison, Angew. Chem. Int. Ed. 2014, 53, 3796.

[2] J. M. Lambert, C. Q. Morris, Adv. Ther. 2017, 34, 1015.

[3] Y. N. Lamb, Drugs 2017, 77, 1797.

[4] E. Perrino, M. Steiner, N. Krall, G. J. L. Bernardes, F. Pretto, G. Casi, D Neri, Cancer Res. 2014, 74, 2569.

[5] M. S. Dennis, H. Jin, D. Dugger, R. Yang, L. McFarland, A. Ogasawara, S Williams, M. J. Cole, S. Ross, R. Schwall, Cancer Res. 2007, 67, 254.

[6] M. Srinivasarao, C. V. Galliford, P. S. Low, Nat. Rev. Drug Discovery 2015 $14,203$.

[7] N. Krall, J. Scheuermann, D. Neri, Angew. Chem. Int. Ed. 2013, 52, 1384.

[8] S. Cazzamalli, A. Dal Corso, D. Neri, Mol. Cancer Ther. 2016, 15, 2926.

[9] S. Cazzamalli, A. Dal Corso, F. Widmayer, D. Neri. J. Am. Chem. Soc. 2018, ASAP, http://dx.doi.org/10.1021/jacs.7b13361. 